\title{
Evolution of the International Metric System of Units SI
}

\author{
W.T. ChYLA* \\ Central Office of Measures, Elektoralna 2, 00-139 Warsaw, Poland
}

(Received June 17, 2011)

\begin{abstract}
Once the New SI is approved by the General Conference on Weights and Measures (CGPM), all base units of the international metric system of units (SI) will be defined in terms of physical constants and atomic properties. In this paper, we consider the rationale and the direction of the possible further evolution of the SI. The idea is to define all base units exclusively in terms of fundamental physical constants, with no reference to specific phenomena, physical theories or properties of material entities (including properties of atoms and elementary particles), so that those definitions would not have to be altered or amended following advancement in our understanding of the structure of matter, emergence of new physical theories or due to the technological progress. New developments in science and technology would then affect only the mise en pratique (realization) of base units, rather than their definitions. Furthermore, we point out the need for including base units for the weak interaction and the strong interaction into the SI and propose a way to do it. The structure of the fundamental-constants-based system of units (the FC SI) is discussed and prerequisites for the implementation of the FC SI are considered. PACS: 06.20.fa
\end{abstract}

\section{Introduction}

There has been a lively debate in recent years on the necessity of replacing the international prototype of the kilogram with a quantum standard of mass [1-6]. The debate, which can be traced back to the 1975 Kibble's concept of a device that is now called the watt balance [7-13] and Resolution 7 of the 21st meeting of the General Conference on Weights and Measures (CGPM) in 1999 [14], turned into a discussion on the need of a comprehensive review of the entire metric system of units (SI). The prevailing opinion is that the upcoming revision of the SI should be restricted to (1) the redefinition of four of its base units, namely the kilogram, the ampere, the kelvin and the mole in terms of physical constants (the Planck constant $h$, the elementary charge $e$, the Boltzmann constant $k_{\mathrm{B}}$ and the Avogadro constant $N_{\mathrm{A}}$, respectively), as suggested in Recommendation 1 of the International Committee for Weights and Measures (CIPM, CI-2005) [15], and (2) rephrasing the other definitions of base units $[16,17]$. The new, coherent, artefact-free system of units, known as the New SI or the Quantum SI, offers so significant benefits to the most advanced areas of metrology and natural sciences, that it seems only a matter of time when it is finally accepted by the whole of the scientific community and approved by the CGPM.

The New SI can be viewed as the latest element in the long sequence of unit systems that evolved toward being entirely free from arbitrarily chosen artefacts. The absence of artefacts in definitions of base units ensures the long-term stability of the unit system, invulnerability to environmental factors (such as fluctuations of tempera-

\footnotetext{
* e-mail: chylawt@wp.pl, address: al. Niepodległości 132/136 m. 30, 02-554 Warsaw, Poland.
}

ture and humidity, surface contamination, cleaning, influence of the natural background radiation, wear due to use of the artefact standards) that might affect properties of primary standards of base units in a way beyond control.

Although the International System of Units (SI) has not been superseded by the New SI yet, it seems worth considering whether the latter is the final say in the area of units of measurement, which will stay with us for all times to come, or there might arise a necessity to rethink the system of units all over again in further future.

This paper advocates the need and the possibility of designing a system of units (or more precisely: the set of definitions of all base units) that is entirely free from any reference to material entities (including atoms and elementary particles) and independent of any specific physical theories (either classical, or relativistic, or quantum). In such approach, material entities, physical phenomena, laws and theories enter the picture only at the stage of the mise en pratique (realization) of base units. This idea stems from the 1983 CGPM decision to define the metre in terms of the speed of light $c$; we claim that it is possible and necessary to define all base units of the SI in terms of fundamental constants.

In the next section, we discuss the contemporary trend in the redefinition of units; it can be viewed as a generalization of the Maxwell idea of atomic standards [18] that employs not only atoms, but also elementary particles as reference standards. The New SI, which defines the ampere in terms of the elementary charge $[16,17]$, can be seen as an example of such a generalization and a precursor of possible further changes.

Section 3 presents motivation for having all base units defined independently of properties of any material entity, including those of elementary particles. This postulate results from the observation that validity of a definition that refers to a material object is unavoidably 
restricted to a certain range of energy, as well as the time and length of the interaction (note that every measurement is a kind of interaction) because every material object is a well defined entity only in a certain spatial, temporal and energy domain. For example, even the basic parameters of elementary particles, such as their rest mass and the (electric) charge, are not uniquely determined beyond a certain range of energy, size and time scale, due to their complex structure.

In Sect. 4, we put forward and discuss the postulate of all base units being defined independently of physical phenomena, theories and laws, as those reflect our current state of knowledge that is subject to change at any time, whereas definitions should be as permanent as possible.

Next, in Sect. 5, we present the structure of the system of units, where all "fundamental base units" are defined exclusively in terms of fundamental physical constants to meet the two postulates mentioned above. The units for the weak interaction and the strong interaction are included in the proposed system of units and their definitions are patterned on the definition of the electric unit to make those definitions harmonized. Such a system of units can be named "the fundamental-constants-based SI", or the FC SI for short. Although no reference to a material entity or a physical law is necessary to define the base units of the FC SI, specific physical relations and material objects may and have to appear in the mise en pratique of those units.

In the final discussion of this paper, we consider the key steps in the historical development of the international system of units (the artefact-based period, the phase of atomic properties serving as reference standards and the era of the physical-constants-based system of units), which positions the FC SI in the final section of the evolutionary chain and examine prerequisites for introducing the FC SI.

Since scientists of different descent (mathematicians, engineers, chemists and purebred metrologists), as well as researchers working in different sub-fields of physics employ diverse terminology, the meanings of certain terms used in this paper have been stated in Appendix, with the intention to avoid any ambiguity.

\section{Maxwell's idea of atomic standards as a driving force behind the contemporary and future evolution of the system of units}

In 1870, J.C. Maxwell noticed [18] that the choice of measurement standards based on macroscopic bodies (either man-made or the Earth) was arbitrary and properties of those standards could change over time. Instead, he proposed to base the system of units on atomic standards that he considered absolute in the sense of atoms being identical and unchangeable, as well as available (at least in principle) to everybody, everywhere and at any time. It is worth noting that Maxwell's idea was truly bold at the time when Dalton's concept of atoms and molecules used to have the status of a working hypothesis, which - although very useful for chemists and for scientists developing the kinetic theory of gases was confirmed experimentally beyond any doubt only at the turn of the 19th and 20th century.

It was not until 1960 that Maxwell's idea was implemented into the international system of units; at that time, the artefact standard of the metre was replaced with the metre defined in terms of the wavelength of a certain spectral line of ${ }^{86} \mathrm{Kr}$ [19]. In today's SI, also the second is defined and realized in terms of the atomic standard (the hyperfine transition in ${ }^{133} \mathrm{Cs}$ ) [20]. The proposed New SI concludes the epoch of the artefact-based system of units, and begins a new era of the artefact-free definitions of base units, as it defines all base units in terms of atomic properties and physical constants $[16,17]$. Actually, the New SI goes even further than the original Maxwell's proposal, because the ampere is to be defined in terms of the elementary charge $e$, which is associated primarily with properties of elementary particles rather than atoms.

As physics and other natural sciences progress, our views on what is absolute vary accordingly. Molecules, once considered identical, turned out to be different due to their different isotopic composition, atomic configuration, conformation of the molecule, optical isomerism, valence differentiation, their electronic, rotational or vibrational energy states, electronic spin state, nuclear spin state, para and ortho variety of electronic and nuclear spin states, nuclear excitation state, uncertainty of mass and energy of short lived excited states, as well as unavoidable effects of environmental interactions, such as polarization by the external electric or magnetic fields and interaction with other molecules that is temperature and pressure dependent. As precision of measurements gradually increases, those effects become large enough to warrant amendments and supplementary provisions that clarify the situation referred to in the definitions of base units. For example, it turned out that the definition of the kelvin required fixing the isotopic composition of water [15]. Also, the reference conditions in the definition of the second (temperature $0 \mathrm{~K}$, fixed rest frame) had to be stated explicitly to enable making corrections for the frequency shift due to ambient radiation; these stipulations have been included in the draft definition of the second in the New SI [17] and a similar amendment concerning local gravity might be in order to account for general relativistic effects. Such modifications are unavoidable where definitions of units are based on material entities, either macroscopic or microscopic.

Less complex physical entities are better suited to play the role of reference standards, because less number of factors can affect their properties. Although atoms and molecules are much less susceptible to the external influences than their macroscopic conglomerates (such as the international standard of the kilogram), they are still quite complex and may have different properties depending on numerous internal and environmental factors, as 
mentioned above. Elementary particles are not only less complex than atoms and molecules, but they are also much less vulnerable to the external influences, because much higher energies are required to affect their properties. Therefore, elementary particles are better suited than atoms to become reference entities when defining units of measurement. The New SI definition of the ampere in terms of the elementary charge $e$ can be considered one example of such a new approach; definition of the kilogram in terms of the mass of an elementary particle (the free electron) has also been proposed [21].

Transition from one type of a standard to another one is a multistage process. For example, let us consider the unit of time, the second. The present definition and the mise en pratique of the second are based on the hyperfine transition in the caesium atom, which occurs at microwave frequencies. Redefinition of the second in terms of electronic transitions of higher frequencies (energies) can improve accuracy and decrease absolute uncertainty in measurements of time. The optical transition in ${ }^{87} \mathrm{Sr}$ and four other optical frequencies (in ${ }^{88} \mathrm{Sr},{ }^{40} \mathrm{Ca}^{+}$, ${ }^{171} \mathrm{Yb}^{+}$and ${ }^{171} \mathrm{Yb}$ ) have already been endorsed by the CIPM in 2009 as valid standard frequencies for realization of the second and the metre [22]. Redefinition of the second in terms of the optical or UV transitions in the hydrogen atom has also been suggested [23], as it is the simplest atomic system whose properties (energy states) can be computed with highest accuracy. If the trend of increasing accuracy of atomic clocks by heightening their frequency persists, the future definition of the second (and the mise en pratique of the second and the metre) might be based on electronic transitions of core electrons in highly ionized heavy atoms (high- $Z$ hydrogen-like ions), which occur in the $\mathrm{keV}$ energy range (X-ray frequencies). The subsequent, yet qualitatively different standard of time would require gamma transitions in atomic nuclei ( $\mathrm{MeV}$ range), but it is difficult to imagine, how the realization of a nuclear clock might look like. It is even more difficult to imagine an elementary-particle-based clock that would operate in the $\mathrm{MeV} \div \mathrm{GeV}$ range of energies (frequencies)* Thus, even though elementary particles can be used as reference standards in the case of some base units (e.g. the ampere in the New SI), this might not be the option for other base units. In the next section, we point out that beyond a certain range of energy, time and length, it might be impossible to use elementary particles as reference standards not only for

\footnotetext{
* Periods of time much shorter than $10^{-15} \mathrm{~s}$ are common in particle physics (e.g. the lifetime of $\Sigma^{0}$ is $\approx 7 \times 10^{-20} \mathrm{~s}$ ), but those values are not measured by direct comparison with the standard of time. Instead, so short periods of time in particle physics are calculated based on relevant physical theories using the values of fundamental constants such as $h$ and $c$ (e.g. from cross-sections for particle decays or from the energy spread in collision experiments) and their accuracy is hardly ever better than $2 \div 3$ significant digits.
}

the lack of an appropriate concept or for technical difficulties, but because of fundamental physical reasons that result from the structure of elementary particles.

\section{Definitions of base units should be free from any reference to material entities}

If a unit is defined in terms of properties of some material entity (either macroscopic or microscopic), or if the definition refers to a material entity in any other way, then validity of the definition is restricted and conditional. Restricted - because it works only in situations where the reference entity exists in the assumed form and shape (i.e. the body must behave as a whole rather than as a collection of its distinct constituent parts). Conditional - because properties of a body, which is used as a reference standard, do change with the range of energy involved in the measurement, size under consideration, temperature and other environmental parameters.

For example, the (historical) international prototype of the metre could be considered a uniform, continuous body as long as its atomic structure could be neglected; at the level of absolute lengths on the order of $10^{-10} \mathrm{~m}$ or less, or $10^{-10}$ in relative uncertainty, such a definition of the metre just fails because of the atomic structure of the international primary standard in the material form, not to mention the environmental effects. Fortunately, the (historical) definition of the metre has been changed well before reaching that level of accuracy.

Material entities that are less complex turn out less sensitive to environmental factors and are better fit to serve as references for measurement units; this is consistent with the trend of moving away from macroscopic standards to atomic standards, and then to standards based on elementary particles and physical constants. However, elementary particles are also complex entities and definitions based on their properties are valid only in situations where the structure of elementary particles can be neglected.

Particles that once were considered elementary and indivisible, later on turned out to be conglomerates of even more elementary entities. Atoms had been considered indivisible elementary building blocks of all matter until experimental works of J.J. Thomson and E. Rutherford proved that they comprise electrons and nuclei. Electrons are still considered elementary particles, while nuclei turned out to be conglomerates of protons and neutrons. In 1964, protons and neutrons, as well as other hadrons, were represented as clusters of more elementary (although inseparable) particles called quarks, bound together by gluons. In 1970s and 1980s there were attempts to build quarks from even more elementary objects (e.g. the rishon model [24]), but that idea has not been confirmed experimentally, at least for now, and it might turn out that the very concept of identifiable particles as separate entities fails at that level of size, lifetime and energy densities. The most "elementary" particles for now (often referred to as fundamental particles) are leptons (which 
includes the electron), quarks and quanta of fundamental interactions (the photon, gluons, $\mathrm{W}^{ \pm}, \mathrm{Z}^{0}$ ), see e.g. [25].

Let us assume that one attempts to use the proton as a reference for some unit of measurement, e.g. the unit of mass or the electric charge. Parameters of such a reference standard are well determined only in situations where the proton does not manifest its internal structure and can be considered indivisible. The proton as a reference fails at distances $l \leq 10^{-15} \mathrm{~m}$, energies $E \geq 1 \mathrm{GeV}$ and in the time range $t \leq 10^{-23} \mathrm{~s}$; in such situations (e.g. in collision experiments) the proton manifests as a conglomerate of constituent particles, each carrying its fractional electric charge, the weak charge and the strong (colour) charge. Besides, mass, momenta and charges of those constituent particles are not uniquely determined (neither the "bare mass" and the "bare charge" of core particles, nor the "dressed mass" and the "dressed charge" of the core particle together with the cloud of virtual pairs); this is the manifestation of the actual physical situation, rather than the problem with accuracy of measurements.

Therefore, one must drop the proton and other compound particles as candidates for becoming absolute standards and reference entities for definitions of units, at least at sufficiently high energies. Instead, one might suggest considering the lightest of massive leptons, the electron, as the reference standard suitable for the definition of the unit of mass or the electric charge. The electron is a point particle which, by definition, does not have any internal structure. Even if the electron is a point particle indeed (which is sometimes questioned by indicating that the other two massive leptons - muon and tauon - can decay into electrons, so that they may turn out to be the excited states of the electron, similarly as the $\Delta^{+}$particle might be interpreted as the "excited state" of the proton) it is still a complex particle: even though the electron does not have the internal structure, it does have the external structure due to vacuum polarization that makes the core of the free electron surrounded by a cloud of virtual particles, mostly electron-positron pairs. In all macroscopic experiments one observes the net electric charge of the electron together with the cloud of its virtual particles (the dressed charge or the electric charge of the dressed electron); the dressed charge manifests also in most of microscopic phenomena. The value of the dressed charge is listed in tables (including CODATA) and it is used in the New SI to define the ampere $[16,17]$. However, the charge of the core of the electron (the bare charge) differs from the dressed charge by about $1.7 \%$, which is calculable with the use of the perturbative QED, see e.g. sect. 4.7, pp. 279-280 in [26] or sect. 7.1 .4 , p. 346 in [27]. This correction comes from the phenomenon often referred to as vacuum polarization. Other effects of the same physical nature, which are of metrological significance, include e.g. the radiative corrections that give appreciable contributions to the Lamb shift and the hyperfine splitting in the caesium atom (see e.g. sect. 2.3.2, pp. 79-82 and sect. 7.3.2 in [27]), the anomalous magnetic moment of the electron (see e.g. sect. 7.2.1, pp. 347-349 in [27]) and many others. Vacuum polarization manifests as a "small" correction in low-energy phenomena, but it is important also at high-energy $\mathrm{e}^{-} \mathrm{e}^{-}$collisions.

A similar problem concerns the rest mass of the electron. The value of the rest mass that manifests in macroscopic phenomena (the one given in tables, including CODATA) is the rest mass of the dressed electron. Its bare mass, which can be computed with the use of the perturbative QED, differs also by about $1.5 \%$ from the dressed mass value (see e.g. sect. 4.7, pp. 268-273 in [26] or sect. 7.1 .2 in [27]).

Since the rest mass and the electric charge of the electron have the uniquely determined values only in a certain (although very wide) range of distances and energies, the electron works fine as the reference entity for the unit of charge (or current) or the unit of mass, but only for a certain class of physical phenomena. Since these two parameters of the electron are not uniquely determined in the whole range of distances and energies, the definitions based on properties of the electron are not unconditional and do not cover the whole range of possible distances, energies and periods of time. In general, if the definition of a unit is based on properties of a point particle, such as the electron, it suffers difficulties of similar nature as the definition based on properties of a compound particle, such as the proton.

The discussion given in this section shows that where definitions of units refer to material entities (no matter how elementary they are), then those definitions do not cover the whole range of possible distances, time periods, energies or frequencies. Besides, definitions that refer (in any way) to certain material entities are bound to evolve (or even undergo a radical change) due to the progress in our understanding of the structure of matter, due to the development of technological capabilities and our research needs. This is certainly not a desirable feature as far as the definition of a unit is concerned; definitions of base units are supposed to be permanent, although their mise en pratique may and should employ material entities and change for scientific and technological reasons. Therefore, a prerequisite for making definitions of base units permanent is that they should be free from references to any material entities.

\section{Definitions of base units should be free from any reference to physical phenomena and physical theories}

Modern physical theories, even though sometimes speculative, are on a par with unquestionable results of experimental physics, because direct observations and measurements are worth only as much as the theory that is used to interpret new experimental findings.

No theory or a specific physical law that has ever existed is immune to changes resulting from new experimental evidence or theoretical considerations. Scientists 
have always been aware that physical laws assume idealized systems so that corrections are necessary to adequately describe the real world situations. Besides, validity of every theory and every physical law is restricted to phenomena occurring in a certain range of spatial magnitudes, in a certain characteristic time, in some range of temperatures and energies of the interaction.

When the scale of the considered system changes by many orders of magnitude and the existing theory fails, then a fundamentally different approach may be necessary; for example, processes in the cosmological scale require general relativistic approach, whereas microscopic phenomena call for the quantum treatment. New theories may change our understanding of fundamental physical notions (such as the spacetime, mass of particles, continuous or discrete quantities) and specific laws (e.g. the law of conservation of energy that failed in the case of radioactive materials). Experience proves that if one tries to apply some well established notions in an entirely new physical situation, he is bound to face their incompatibility, e.g. the failure of the notion of simultaneity of events in relativistic situations or the particle-wave duality in the quantum world. A new physical realm has to be handled in an entirely new way. Nevertheless, new physical theories generalize (i.e. broaden the range of validity) rather than invalidate the theories they supersede. For example, the QED does not invalidate Maxwell's electrodynamics in classical situations; general relativity can be reduced to the Newtonian gravity in weak local fields; the Ehrenfest theorem provides a link between the classical and quantum mechanics.

The definition of a unit that is based on a certain physical effect and is described in the framework of a certain theory, may imply different values of the unit at different levels of our understanding of that phenomenon. One example is the present SI definition of the ampere. It was adopted in 1948 and it is based on the assumption that Maxwell's equations are perfectly exact. At approximately the same time, a new theory emerged, the QED, which implies that the force of the electromagnetic interaction between two bodies is somewhat different from the value yielded by classical electrodynamics due to the quantum nature of the electromagnetic field ${ }^{\dagger}$. Therefore, the present SI definition implies somewhat different values of the ampere for one who works in the QED regime and for one who applies the classical approximation. This discrepancy would not occur if the value of the ampere were defined by fixing the value of $\mu_{0}$ (or $\left.\varepsilon_{0}\right)$ explicitly, with no direct or indirect reference to the force of interaction between two parallel linear conductors and its theoretical description.

\footnotetext{
$\dagger$ Recall the Casimir effect that predicts a nonzero force of the electromagnetic attraction between two electrically neutral parallel plates, which is inversely proportional to the fourth power of separation between the plates, whereas the classical electrodynamics gives a null result, see e.g. [28-30] and sect. 3.2.4 in [27].
}

One might say that such minor effects are of no practical significance; however, what is a subtle matter of principle today, it may be of practical importance at higher levels of accuracy or in a different kind of a system (scaling fails). For example, quantization of the electromagnetic field may be negligible in measurements of the current in macroscopic systems at the present level of accuracy ${ }^{\ddagger}$, but this is not the case in microscopic systems, such as nanostructures, where the fully quantum treatment might be necessary.

The proposed redefinition of the ampere in terms of the elementary charge $[16,17]$ alleviates this problem, because the new definition of the ampere is insensitive to a particular theory of the electromagnetic field; yet, the proposed definition requires clear distinction between the elementary charge $e$ (a constant by definition) and the effective charge of elementary particles at very high energies due to their complex structure.

To make things short, no theory or a physical law will ever provide the ultimate insight into the workings of Nature; physical laws did, do and will evolve, as they reflect our current and temporary state of knowledge. If definitions of base units are to be permanent, they cannot be based on specific phenomena and physical theories of the present, because those theories are bound to change, which would affect the value of the unit, and it would imply the necessity of repeated alterations of definitions of units. To make definitions of base units permanent, they should be free from any references to physical phenomena, theories and laws, either classical, or quantum, or relativistic. Progress in theoretical and experimental science, expressed in the form of new physical phenomena and theories, should manifest via the evolution of the mise en pratique of those units.

\section{The system of units where all base units are defined exclusively in terms of physical constants (FC SI)}

According to the discussion in the previous two sections, let us postulate that definitions of all base units should be free from any reference to material entities, physical phenomena and physical theories. This section describes the structure of the system of units (FC SI) which satisfies this postulate, as all its fundamental base units are defined exclusively in terms of fundamental physical constants.

\subsection{Fundamental base units of the FC SI}

Fundamental base units are such that: (1) they correspond to all fundamental notions of physics (i.e. primary and indefinable notions, such as time, length, mass and

\footnotetext{
$\ddagger$ Presently, the most accurate measurements of the current employ the Josephson effect and the quantum Hall effect, rather than the definitional realization of the ampere.
} 
quantized charges), in terms of which all other observable quantities can be expressed, at least in principle; (2) they can be defined exclusively in terms of fundamental physical constants, i.e. $c, h, G$ and coupling constants of quantized interactions (see Appendix); (3) since all (derived) physical quantities can be expressed in terms of time, length, mass and charges, their numerical values can, in principle, be expressed exclusively in terms of fundamental base units (the second, the metre, the kilogram and units of quantized charges), although other units may be introduced for convenience of users; (4) the number of fundamental base units cannot be reduced without compromising uniqueness of their physical meaning (which might happen, e.g. where a distance is expressed in terms of the unit of time).

Table I gives definitions of fundamental base units that correspond to fundamental physical quantities (time, length, mass and charges). The definitions are expressed exclusively in terms of fundamental physical constants $c$, $h, G$ and coupling constants of quantized interactions, which play the role of generators of fundamental base units of the FC SI. The Xs in parentheses stand for one or more additional digits, as in the Draft [17]; the Xs shall be adjusted to assure that the redefinition of units will not cause appreciable changes in the values of units that could affect the laboratory practice.

Fundamental base units of the FC SI.

TABLE I

\begin{tabular}{|c|c|c|c|}
\hline Quantity & Unit & Symbol & Definition \\
\hline Time & second & $\mathrm{s}$ & \multirow{3}{*}{$\begin{array}{l}\text { The second, the metre and the kilogram, which are the units of time, length and } \\
\text { mass, respectively, are such that the speed of light in a vacuum is exactly } c= \\
299792458 \mathrm{~m} \mathrm{~s}^{-1} \text {, the Planck constant is exactly } h=6.62606(\mathrm{X}) \times 10^{-34} \mathrm{~kg} \mathrm{~m}^{2} \mathrm{~s}^{-1} \\
\text { and the gravitational constant is exactly } G=6.6742(\mathrm{X}) \times 10^{-11} \mathrm{~kg}^{-1} \mathrm{~m}^{3} \mathrm{~s}^{-2} \text {. }\end{array}$} \\
\hline Length & metre & $\mathrm{m}$ & \\
\hline Mass & kilogram & $\mathrm{kg}$ & \\
\hline $\begin{array}{l}\text { Electric } \\
\text { charge }\end{array}$ & coulomb & $\mathrm{C}$ & $\begin{array}{l}\text { The coulomb, the unit of the electric charge is such that the electromagnetic } \\
\text { coupling constant is exactly } k_{\mathrm{e}}=\left[4 \pi \times 8.85418(\mathrm{X}) \times 10^{-12}\right]^{-1} \mathrm{C}^{-2} \mathrm{~kg} \mathrm{~m}^{3} \mathrm{~s}^{-2} \text {. }\end{array}$ \\
\hline $\begin{array}{l}\text { Weak } \\
\text { charge }\end{array}$ & $\begin{array}{c}\text { no name } \\
\text { weinberg? }\end{array}$ & $\begin{array}{l}\text { no name } \\
\text { W? }\end{array}$ & $\begin{array}{l}\text { The [weinberg?], the unit of the weak charge is such that the weak coupling } \\
\text { constant is exactly } k_{\mathrm{w}}=(\mathrm{X}) \text {. }\end{array}$ \\
\hline $\begin{array}{l}\text { Strong } \\
\text { charge }\end{array}$ & no name & no name & $\begin{array}{l}\text { The [no name], the unit of the strong charge is such that the strong coupling } \\
\text { constant is exactly } k_{\mathrm{s}}=(\mathrm{X}) \text {. }\end{array}$ \\
\hline
\end{tabular}

The units of time, length and mass in the FC SI are established simultaneously, in a single definition, which puts the second, the metre and the kilogram on equal footing. This is quite different from clear seniority of base units in the present SI and in the New SI, where the unit of time is (implicitly) defined first, then the second is explicitly used to define the metre, and finally (in the New SI) the new definition of the kilogram makes use of the previously defined units of time and length. Formally, this feature of the FC SI results from the fact that $h, c$ and $G$ are associated with at least two of the three fundamental physical notions; in other words, there are no fundamental constants that could have been associated exclusively with time, or distance, or mass. In physical terms, this is the manifestation of the deep connection between these notions in the physical realm: space and time are two different aspects of a single physical entity, the spacetime, which has first been noticed in the special relativistic regime; the interconnections between all three notions (space, time and mass) manifest clearly in the general relativistic realm. We need to recognize that our macroscopic world, where we have got used to treat the space, time and mass as separate entities, independent of each other, is just the weak field approximation of the actual, general relativistic situation, where these three notions are (in general) inseparable. The physical dimensions of fundamental physical constants $c, h$ and $G$ reflect that relationship and this implies that the corresponding fundamental base units (s, m, kg) must be defined simultaneously (otherwise, the first definition would have referred to a previously undefined unit). This feature of the FC SI should be interpreted as a manifestation of the profound physical relation between space, time, and mass, rather than an inconvenience of no physical significance.

It should be noted that the possibility of defining all (seven) SI base units simultaneously, without ascribing a particular constant to a particular unit, with the option of including the 22 SI derived units into a single definition, has been presented in [16], sect. 2.4. Such approach, however, would have mixed the three fundamental base units of the SI ( $\mathrm{s}, \mathrm{m}$, and $\mathrm{kg}$ ), which ought to be defined simultaneously for clear physical reasons, with the ampere, the other three base units of the SI (the kelvin, the mole and the candela) and numerous derived units of a clearly different physical status, and that should be avoided.

One might wonder why the gravitational coupling constant $k_{\mathrm{g}} \equiv G$ is used differently, compared with the other coupling constants of fundamental interactions 
$\left(k_{\mathrm{e}}, k_{\mathrm{w}}, k_{\mathrm{s}}\right)$ to generate fundamental base units; should not all the coupling constants be used in an analogous way to define units? There are important physical reasons for the difference: (1) in general, mass is the element of the spacetime metric, so that mass and gravity is associated with global properties of spacetime, whereas charges are essentially local notions; (2) mass changes continuously in the special relativistic realm, whereas the electric charge (and the other charges) are velocity-independent, as they are the Lorentz invariants; (3) mass is the element of covariant and contravariant quantities (e.g. the energy-momentum vector, the energy-stress tensor) that transform quite differently from the charges, which are true scalars; (4) the gravitational interaction cannot be quantized (as for now), whereas the electric charge, the weak charge and the strong charge are intrinsically quantized entities and their interactions have the quantum nature. Gravitation is the kind of interaction entirely different from the other fundamental interactions (as of now) and it is not surprising that there is no symmetry in the way the $G$ and the coupling constants $k_{\mathrm{e}}, k_{\mathrm{w}}, k_{\mathrm{s}}$ are used to define units.

The definition of the coulomb in this paper makes use of the fixed value of the electromagnetic coupling constant $k_{\mathrm{e}}=1 /\left(4 \pi \varepsilon_{0}\right)$. Instead of fixing the $k_{\mathrm{e}}$, one might fix the value of the electric constant $\varepsilon_{0}$ (permittivity) or the magnetic constant $\mu_{0}$ (permeability); fixing the value of the elementary charge $e$, as in the New SI, is also a possibility (see Appendix). In any case, units of the three quantized charges should be defined in an analogous manner.

In principle, one could choose either the coulomb or the ampere as the base electric unit; the coulomb has been chosen here as the base unit because (1) the electric charge, rather than the current, is the primary notion from the physical point of view; (2) the choice of the ampere as the base unit would have involved the second in the definition of the electric unit, which would create the unnecessary interdependence between those units at the level of the definition; (3) definitions of units for all three kinds of quantized interactions should be structured analogously (harmonization); the notion analogous to the electric current does not make sense in the case of the weak and the strong charge (the QCD colour currents have entirely different meaning).

The present SI definition of the ampere, which implies the exact value of the magnetic constant $\mu_{0}$ (and consequently the exact value of $\varepsilon_{0}$ and $k_{\mathrm{e}}$, due to the relation $c^{2}=\varepsilon_{0}^{-1} \mu_{0}^{-1}$ ), is supposed to be succeeded by the New SI definition of the ampere in terms of the elementary charge $e$; therefore, the return to the $\mu_{0}$ (or, equivalently, to the $\varepsilon_{0}$ or $k_{\mathrm{e}}$ ) in the FC SI might seem a step backwards. Actually, the two options are equivalent: (1) one can fix the value of the elementary charge $e$ (treated as the coupling constant, as most particle physicists actually do) in the definition of the electric unit and consider $\varepsilon_{0}, \mu_{0}$, and $k_{\mathrm{e}}$ as quantities to be measured; or (2) one can fix the value of $\varepsilon_{0}$ or $\mu_{0}$ or $k_{\mathrm{e}}$ (treated as the coupling constant) in the definition of the electric unit (coulomb) and consider the elementary charge $e$ as a quantity to be measured. In both situations one needs to remember that (1) one cannot fix the numerical values of the $e$ and $\varepsilon_{0}$ (or $\mu_{0}$ or $k_{\mathrm{e}}$ ) simultaneously, neither explicitly nor implicitly, because it would lead to inconsistency; (2) the elementary charge $e$ is not the same as the electric charge of any specific elementary or fundamental particle, due to vacuum polarization, as mentioned in Sect. 3; (3) if the electric charge of a particle is written as $Q=q e$, where $q$ is the charge of the particle in units of $e[\mathrm{C}]$, then $q$ is not necessarily an integer.

The advantage of fixing the value of $e$ (in the New SI definition of the ampere) is that it gives exact values of numerous important physical constants, such as the von Klitzing constant, the Josephson constant, the Faraday constant, the Stefan-Boltzmann constant, etc., and it seems to be a decisive factor at this time. On the other hand, the second option is physically more advantageous in further future, as it allows distinguishing the notion of the electric charge from the notion of the electromagnetic coupling constant, just as the notion of mass is clearly distinguished from the gravitational coupling constant, even though the two quantities go together as a product. The same concerns the weak charge and the strong charge and their coupling constants.

Vacuum polarization concerns also the weak charge of quarks and leptons and the strong (colour) charge of quarks. Fundamental particles are surrounded by very short-ranged clouds of virtual particles that screen their weak and/or strong charge, which affects both their effective charge and their effective mass. The screening effect makes the effective (dressed) charges, weak and strong, seem smaller at larger distances from the core (bare) particle; the effect, known as the "running of charges", has been established experimentally both for the weak and the strong charge [31, 32]. For this reason and in analogy to the electromagnetic interaction, it seems natural to choose the coupling constant of the weak interaction $k_{\mathrm{w}}$ and the coupling constant of the strong interaction $k_{\mathrm{s}}$ as generators of units of the weak charge and the strong charge, respectively. It is up to experimental particle physicists to provide values for the coupling constants and to give names to units of those charges. Once the values of the two coupling constants, $k_{\mathrm{w}}$ and $k_{\mathrm{s}}$, are fixed by definitions of the corresponding units, the weak charge and the strong charge of elementary particles become measurable quantities.

On the other hand, in analogy to the New SI approach to the electromagnetic interaction, one can choose the "elementary weak charge" and "the elementary strong charge" as the coupling constants of the weak and the strong interaction, respectively, and ascribe (by definition) certain values to those quantities. Then (1) the $k_{\mathrm{w}}$ and $k_{\mathrm{s}}$ would become measurable quantities (no longer in a status of coupling constants); and (2) weak and strong charges of fundamental particles would become measurable quantities that are not necessarily integer multiples 
of the corresponding elementary charges. Whatever the choice, there should be symmetry in the formulation of definitions of units for all three kinds of quantized interactions (harmonization). As long as the SI does not attempt to define units for the weak and the strong interaction, the choice is irrelevant.

Inclusion of the weak and the strong interaction into the SI is one thing, and the consequence of the unification of interactions on the system of units is a separate problem. The unification can be understood as (1) the unified description of fundamental quantized interactions (this includes the electroweak theory, which is complete, and the unification of the electromagnetic, weak and strong interactions, i.e. the Standard Model, which, although not a closed theory yet, is not seriously challenged by other theories); or (2) unification at the GUT scale of energies, with the gravitational interaction included, where all four interactions might merge into one interaction, with a single value of the coupling constant. Any considerations on consequences of the latter type of the unification on the system of units would be premature. Unification of gravity with quantized fundamental interactions would require rethinking the fundamental notions of physics and the corresponding units, which is not the subject of this paper.

Unification of the electromagnetic and the weak interaction does not change much in the area of units, compared with the two interactions treated as independent ones. Although the electromagnetic coupling constant and the weak coupling constant (i.e. the elementary electric charge and the elementary weak charge, using the particles and fields terminology) are linearly proportional, see e.g. equation 21.3.19 in [33], the number of independent constants does not change, because the relation between the two coupling constants involves a new constant quantity, the Weinberg angle, $\theta$.

Considering the unification, it might seem appropriate to define the two unit charges (the coulomb and the wein- berg?) simultaneously by fixing the values of the corresponding coupling constants (i.e. $k_{\mathrm{e}}$ and $k_{\mathrm{w}}$, as proposed in this paper) in a single definition. When the Standard Model is a closed theory, the units for the electromagnetic, weak and strong interactions ought to be defined by fixing values of the corresponding three coupling constants $\left(k_{\mathrm{e}}, k_{\mathrm{w}}\right.$ and $k_{\mathrm{s}}$, using this paper's terminology) simultaneously, in a single definition.

One important advantage of defining units in terms of fixed values of physical constants, pointed out in the discussion of the New SI [16], is that macroscopic and microscopic quantities of the same kind (e.g. the mass) are linked with each other directly via those physical constants, so that there is no need to transfer the unit of a given quantity over many orders of magnitude, and that protects against the high increase in the relative uncertainty. For example, when mass of a macroscopic body is determined with the use of the watt balance (which relates the value of mass to $h$ ) and the mass of the electron is also expressed in terms of the Planck constant, then those masses can be compared directly, with no intermediate steps, even though both values differ by some 30 orders of magnitude; this ensures consistent use of the unit of mass in the whole spectrum of magnitudes. The same holds true for the FC SI except for there is no one-to-one correspondence between the unit of mass and the Planck constant (as in the New SI), as the second, the metre and the kilogram are defined in terms of three fundamental constants $(c, h$ and $G$ ) simultaneously and on equal footing.

\subsection{Supplementary base units of the FC SI}

The other two base units of the FC SI and their definitions are given in Table II. The kelvin and the mole stay as base units in the FC SI, because they are very convenient and commonly used, although one could do without both of them.

Supplementary base units of the FC SI.

TABLE II

\begin{tabular}{c|c|c|l}
\hline \hline Quantity & Unit & Symbol & \multicolumn{1}{c}{ Definition } \\
\hline Temperature & kelvin & $\mathrm{K}$ & $\begin{array}{l}\text { The kelvin, the unit of temperature, is such that } \\
\text { the Boltzmann constant is exactly } k_{\mathrm{B}}=1.3806(\mathrm{X}) \\
\mathrm{kg} \mathrm{m}^{2} \mathrm{~s}^{-2} \mathrm{~K}^{-1} .\end{array}$ \\
\hline Amount of substance & mole & $\mathrm{mol}$ & $\begin{array}{l}\text { The mole, the unit of the amount of substance, is } \\
\text { such that it contains exactly the Avogadro number } \\
N_{\mathrm{A}}=6.02214(\mathrm{X}) \text { of particles of a specified entity. }\end{array}$
\end{tabular}

Temperature characterizes the average thermal energy of particles in a certain ensemble of particles in the state of thermodynamic equilibrium. Therefore, in principle, one could express this quantity in terms of the unit of energy, $\mathrm{kg} \mathrm{m}^{2} \mathrm{~s}^{-2}$; this is actually practiced in many fields of physics, especially in statistical physics, where it is quite common to use $\Theta=k_{\mathrm{B}} T$ (see e.g. sect. 5.3, p. 99 in [34]) or $\beta=1 / k_{\mathrm{B}} T$ (see e.g. $[34,35]$ ) as a measure of thermal energy, instead of temperature $T$ measured in kelvins. This, however, would be inconvenient in the macroscopic world, in thermodynamics and also in statistical physics, because the relation between temperature 
and the total thermal energy of particles is different for different kinds of physical entities (e.g. monatomic, linear or nonlinear molecules, for bosons or fermions), in different states of matter (gas, liquid, solid) and at different levels of excitation of internal (vibrational, rotational) degrees of freedom. Secondly, temperature deserves its "own" unit because it is one of the most important and the most often measured variables (or parameters, depending on the situation). Thirdly, as the New SI shows $[16,17]$, the unit of temperature can be precisely and elegantly defined in terms of the Boltzmann constant, and that approach has been adopted in the FC SI.

The qualifier "thermodynamic" in relation to temperature has been purposely omitted in the definition of the kelvin, because it is high time to identify the thermodynamic temperature (defined in terms of macroscopic phenomena) with its microscopic (statistical) counterpart: once $k_{\mathrm{B}}$ and $N_{\mathrm{A}}$ are fixed by definitions, the statistical measure of thermal energy $k_{\mathrm{B}} T$ is entirely equivalent to its macroscopic counterpart, $R T=N_{\mathrm{A}} k_{\mathrm{B}} T$. Although various kinds of temperature do appear in physics (e.g. the spin temperature or negative absolute temperature in the situation of population inversion in lasing systems) the notion of temperature is so well and uniquely determined both in thermodynamics and in statistical physics that it does not need any special qualifiers to specify what one has on mind. In this context, it is worth recalling major research attempts undertaken in 1960s and 1970s to distinguish between the inertial mass, the active gravitational mass and the passive gravitational mass, which however led to no significant results.

The mole is just the traditional and convenient measure of a macroscopic number of microscopic entities of a specified kind. We could, in principle, give up using moles, but then the numerical factor on the order $10^{23}$ would occur in many of the chemical and physico-chemical formulae. Besides, the New SI shows [16, 17] that the mole can be precisely and elegantly defined in terms of the Avogadro constant, and that concept has also been adopted in the FC SI.

The definition of the mole ought not to state possible kinds of chemical and physical entities [17] because (1) the definition is not the proper place to make an explicit list of all possible kinds of particles (if new kinds of particles are discovered, should the definition of the mole be amended?); (2) such a list does not have a chance to be complete anyway (what about free radicals, skyrmions, axions, the Higgs bosons, other fundamental and elementary particles and their supersymmetric partners?); (3) it would be inconsistent to single out some particles (e.g. the electron) and forget about the other ones (e.g. the proton [17]); (4) the choice of criteria according to which a certain entity is (or is not) considered as being of a given kind should be left to the user, as exemplified below: (a) from the optical/spectral point of view, molecules of different conformations or in different excited states are different physical entities, while those molecules can be indistinguishable in chemical reactions; (b) large organic dye molecules in the neutral or in the ionic form can be categorized as the same dye molecule, while they can be considered as entirely different entities from the viewpoint of their staining properties; (c) protons, hydrogen atoms and hydrogen molecules are certainly entirely different kinds of particles for most scientists, but not for those who investigate overall abundances of chemical elements in the interstellar gas.

The notable omission in Table II is the candela, which is shifted to the category of descriptive units given in Table III. The unit of luminous intensity in the SI is defined in terms of a single, specified frequency, chosen quite arbitrarily, which does not characterize the source of light sufficiently, neither from the physical point of view nor from the viewpoint of physiological properties of the human eye. The importance of the candela results from the role it plays in the lighting industry, but that is not a sufficient reason to keep it as the base unit. Recent developments in the lighting industry (i.e. the widespread use of LEDs) might stimulate a comprehensive review of units in optics.

\subsection{Other categories of units in the FCSI}

The core of the FC SI is the system of fundamental and supplementary base units and their definitions. All other units can be sorted out into two categories: descriptive units and compound units, as shown in Table III below.

Other categories of units within the FC SI.

TABLE III

\begin{tabular}{c|l|l}
\hline \hline Category & \multicolumn{1}{|c}{ Examples } & \multicolumn{1}{c}{ Criterion } \\
\hline Descriptive units & $\begin{array}{l}\text { candela, hertz, } \\
\text { dioptre, sievert, } \\
\text { gray, katal }\end{array}$ & $\begin{array}{l}\text { Units that are not uniquely defined by a combination } \\
\text { of base units, and require an operational definition or } \\
\text { a specific comment. }\end{array}$ \\
\hline Compound units & $\begin{array}{l}\text { newton, volt, farad, } \\
\text { gray/second, } \\
\text { katal/metre }\end{array}$ & $\begin{array}{l}\text { Any combination of base units and/or descriptive } \\
\text { units with a numerical factor of 1 exactly that does } \\
\text { not require any comment. }\end{array}$
\end{tabular}


Descriptive units are those that require some kind of additional information to be properly understood. The information may concern (1) geometrical arrangement (e.g. the radian defined in terms of the length of the circular arc and the radius of curvature); (2) the measurement setup (e.g. where the $\mathrm{mmHg}$ is used as a unit of pressure); (3) properties of the material (e.g. the litre is the unit of volume of materials that have the volume elasticity, but not the shape elasticity); (4) the kind of a phenomenon (e.g. the hertz $=\mathrm{s}^{-1}$ can be used only in the context of periodic phenomena, whereas the becquerel $=\mathrm{s}^{-1}$ is the unit of activity of radionuclides, which is a non-periodic effect); (5) a special area of application (e.g. the dioptre $=\mathrm{m}^{-1}$ is used in optics of lenses and mirrors, while $\mathrm{m}^{-1}$ or $\mathrm{cm}^{-1}$ are still used in the infrared spectroscopy); (6) special physical arrangements (e.g. the electronvolt); (7) special chemical or biological processes (e.g. the katal); or (8) specific situations where it is valid to use the unit (e.g. the day, the astronomical unit, the hectare, the bar, the knot).

One can think of many other situations, which require specific additional information to make the unit defined in a precise and unique way or to indicate the context in which the unit can be used. Many units that are already in use or are being developed in chemistry, in food industry, in life sciences, biophysics, medicine and related sciences are designed to satisfy the needs of users and cannot be traced directly and unconditionally to the fundamental physical notions, because (1) the units are relevant to measurements where environmental parameters cannot be precisely controlled (e.g. the chemical composition of samples in pharmacology and in medical diagnostics); or (2) apply to human senses, such as taste or smell. Such units belong to the category of "descriptive units", whose population is expected to grow together with metrology covering ever new areas.

Compound units are all those that are created by multiplication or division of previously defined units and do not require any additional information to be uniquely defined and understood. Compound units can be built of base units and descriptive units.

From the physical point of view, the two "supplementary base units" (the kelvin and the mole) might be reckoned among "descriptive units"; then, one might prefer to recognize only two categories of units: "the fundamental base units" and "the other units". Different viewpoints on how to categorize all those "other" units are welcome. However, the proposal to entirely renounce categorizing of units, which appeared as a spin-off concept in the discussion on the New SI, would go against the necessary distinction between a few truly fundamental physical notions (time, length, mass and charges) and their units versus a multitude of other quantities and their units designed for convenient description of the physical world.

The units for "fundamental physical notions" can be compared to the finite set of basic letters on the keyboard $(\mathrm{a}, \mathrm{b}, \mathrm{c}, \ldots)$, which are the building blocks of the countless number of words (units of derived quantities). The units of "supplementary base notions" are like diphthongs that have been ascribed specific symbols (e.g. æ) and with common consent - added to the basic keyboard for convenience of users, although those symbols could be expressed in terms of basic letters (e.g. $æ=$ ae). The units of "descriptive notions" are like ç, å or ñ that express local ethnic needs to build even more words (more units useful in special fields of science); those ethnic letters are not added to the basic keyboard and require additional information on how to type (use) them. All accepted combinations of those letters (all units) make the vocabulary (the SI). A collection of all accepted combinations of letters is not sufficient to communicate. However, if one ascribes the meanings (values) to the combinations of letters in the vocabulary and adds the rules of grammar (the physical laws) he can write a novel that describes the Universe.

\section{Discussion}

\subsection{A comprehensive view on the evolution of the international system of units}

The international metric system of units came into being with signing of the Metric Convention in 1875, which sanctioned the international standards of mass and length in the form of artefacts. The international prototypes of the kilogram and the meter were deposited at the BIPM; their copies were prepared, selected and distributed among the participating states by the end of 1889. The unit of time has not been the subject of the Metric Convention, because the traditional definition of the second as $1 / 86400$ th part of the mean solar day seemed to be sufficiently accurate and commonly accepted at that time.

The international standard of length remained in the form of the artefact until 1960, when the International System of Units (the SI) was voted and approved by the CGPM. The SI of 1960 defined the unit of length, the meter, in terms of the wavelength of a certain spectral line of ${ }^{86} \mathrm{Kr}$. Although the origin of this definition can be traced back to the 1881 interferometric experiment of Michelson and the 1892-93 Michelson-Benoît measurements of the red spectral line of Cd, the CGPM decision of 1960 was the first formal implementation of the proposal put forward by Maxwell [18] to base the international system of units on atomic standards. A few years later, in 1967/68, the CGPM decided to express the unit of time in terms of the atomic standard as well; the second has been defined in terms of frequency of the hyperfine transition in the ${ }^{133} \mathrm{Cs}$ atom.

The next milestone in the development of the system of units was the 1983 CGPM decision on defining the unit of length in terms of the fundamental physical constant, the speed of light $c$. The metre has been defined as the length of the path travelled by light in a vacuum in a stated time interval.

From 1983 till now, the base units of the SI are defined in three fundamentally different ways: the kilogram is 
defined in terms of the artefact (as the mass of the international prototype of the kilogram), the second is defined in terms of the atomic standard $\left({ }^{133} \mathrm{Cs}\right)$, and the metre is defined in terms of the fundamental physical constant (the speed of light, $c$ ). The above summary highlights the evolution of definitions of base units according to the following scheme:

$$
\begin{aligned}
& (\text { Artefact standards }) \rightarrow \text { (Atomic standards }) \\
& \quad \rightarrow \text { (Fundamental-constants-based standards })
\end{aligned}
$$

with many intermediate steps in between and considerable temporal overlap of those three types of approach.

Presently, we are facing the next essential change in the international system of units, subject to the CGPM approval. The expected redefinition of the kilogram in terms of the Planck constant $h$ (beside redefinitions of other base units) will eliminate the last artefact (the international prototype of the kilogram) from the set of definitions of base units, so that the new system of units will refer exclusively to atomic properties and physical constants. Therefore, the new international system of units, often called the New SI or the Quantum SI, might also be named "the Artefact-Free SI" (AF SI for short), if proponents of the New SI agree. The advantage of the proposed name is that one should expect ever newer versions of the New SI (e.g. the unit of time can be redefined in terms of spectral lines of ever higher frequencies, and the new value of the second will affect values of other base units) and all those successive versions of the New SI will belong to a single distinct class of the Artefact-Free SI.

Where a base unit is defined in terms of atomic properties, the definition will inevitably show its shortcomings some time in future. Consider the second defined in terms of a spectral transition: (1) frequencies of spectral transitions are never exact, not only because of such effects as interaction broadening, the Doppler effect or the resolution of measuring instruments, but also for fundamental physical reasons, such as the Heisenberg principle (uncertainty principle) and the finite lifetime of excited states, which cannot be overcome by technical means. Limited accuracy is acceptable as long as the mise en pratique of units is concerned, but it should not be inscribed into definitions of units. (2) If the unit of time is defined in terms of frequency of a certain spectral line, it is bound to change some time later, because higher frequency reference spectral lines allow for higher accuracy. The mise en pratique may and should evolve together with the scientific and technological progress, but the definition should be permanent. (3) A change in the definition of the second would affect also the other units because of the hierarchic structure of the SI and the New SI; such consequential effects should be avoided. (4) Accuracy of measurements of time is so high $\left(\sim 10^{-16}\right.$ in relative uncertainty) that it requires taking into account the general relativistic effects. Therefore, the definition of the second in terms of an atomic clock requires an explicit statement on: (a) the kind of time they measure, i.e. the "local standard time", using the general relativistic ter- minology (as compared to other general relativistic time scales and the corresponding clocks, e.g. the "coordinate time" and the "coordinate clock"; and (b) the procedure to compare indications of clocks positioned at different locations, where the $g_{00}$ element of the spacetime metric has different values, which is not a trivial problem (see sect. 4 in [36]).

It must be noted here that the discussion in the above paragraph should not be understood as a criticism of the present methodology of defining the second, because it is the only feasible approach at this time. The above discussion, just as the whole paper, presents the rationale for possible future changes (beyond the New SI) in the international system of units and indicates the direction of the expected evolution of the SI in further perspective.

According to the point of view advocated in this paper, the base units ought to be defined independently of physical properties of material entities (including atomic and elementary particle systems) and should not refer to physical phenomena and physical theories, so that the definitions can be free from limitations imposed by material characteristics, technical capabilities and our present (i.e. temporary) state of knowledge. The proposed FC SI satisfies these postulates by defining all fundamental base units in terms of fixed values of fundamental physical constants. This route, however, will be possible only in further future because the FC SI requires much higher accuracy in measurement of $G$ than it is presently possible.

\subsection{Requirements for the implementation of the FC SI}

Implementation of the FC SI is a matter of further future because it requires the gravitational constant $G$ being measured with relative uncertainty of $\sim 10^{-8}$ (in analogy to the requirement imposed on $h$ to redefine the kilogram), whereas the relative uncertainty of $G$ currently cited by CODATA is $1.0 \times 10^{-4}$, only 2 orders of magnitude better than the Cavendish result of 1798 . Measurements of the gravitational constant are very demanding because forces of gravity in laboratory situations are extremely weak and even tiny disturbances may significantly affect the final result [37]. However, before 1990 accurate determination of $G$ did not attract as much attention as precise measurements of other fundamental constants and there is high potential for improvement in this area. The review of research in this field before 1998 can be found in conference materials [38] and more recent references are given in [39]. Let us only mention that traditional methods of measurement of $G$ approach relative uncertainty $10^{-5}$ (see e.g. $[40,41]$ ) and there are theoretical estimates that push the relative uncertainty below $10^{-7}$ in precision tracking of two test bodies performing relative motion in space [42]. It is also worth mentioning the atom interferometry method, which showed significant progress in recent years [43, 44], especially because it is based on a quantum phenomenon that is sensitive to the gravitational field and dependent explicitly on the gravitational coupling constant $G$ (i.e. such a design may be considered a quantum standard of gravity). The possibility of implementing a system of units based exclusively 
on fundamental physical constants might provide strong motivation for precise measurements of $G$.

It seems that the technical aspect (the ability to measure $G$ with sufficient accuracy) may not be the decisive factor in launching the FC SI, because the possibility of making significant amendments to the system of units depends heavily on the human factor. The FC SI is even more abstract than the New SI and consequently it might be difficult to achieve immediate consensus on the new definitions of base units and their mise en pratique. Let us recall that it took 79 years, i.e. three generations, from the time of the first interferometric measurements of length by Michelson (1881) to the CGPM approval of the SI definition of the metre in terms of the wavelength of a certain reference spectral line (1960). So much time was necessary to attain widespread understanding of the physical principle, develop the technology convenient for use in laboratories that did not aspire to perform the top-level research, gain the necessary expertise in all laboratories concerned, and make the measuring system available to all interested laboratories at affordable prices.

Another precondition for introducing the full version of the FC SI is the determination of $k_{\mathrm{w}}$ and $k_{\mathrm{S}}$ with relative uncertainty $\sim 10^{-8}$. Although one can imagine the temporary version of the FC SI that does not include units for the weak and the strong interactions, implementation of the FC SI will still be a true challenge and various versions of the New SI will stay with us for many decades (taking for granted that the New SI is soon accepted).

Contrary to the FC SI that is aimed at the target solution, the New SI demonstrates a pragmatic approach: (1) it concentrates on what is possible presently or in near future (the watt balance); (2) the set of constants that define base units in the New SI is chosen according to the preponderance of advantages at a given time and it is expected to change depending on theoretical developments and the technological progress $[45,46]$. The idea of the FC SI allows to view the New SI in a broad context of not only the past changes and present capabilities, but also from the perspective of possible future developments, which shows that the New SI is a true milestone in a long path toward the enduring system of units.

\section{Appendix}

\section{A note on terminology adopted in this paper}

There is a variety of opinions on how many and which physical constants are truly fundamental [47]. In this paper, fundamental (physical) constants are those that are indispensable to account for elemental physical phenomena. These are: the speed of light $c$ (relativistic phenomena), the Planck constant $h$ (quantum phenomena), the coupling constant of the gravitational interaction $G$ (gravity) and as many (other) coupling constants as many quantized interactions are to be taken into account. At this point it seems that the electromagnetic coupling constant $k_{\mathrm{e}}=1 /\left(4 \pi \varepsilon_{0}\right)$, or any equivalent constant such as $\varepsilon_{0}$ or $\mu_{0}$, one constant associated with the weak interaction $\left(k_{\mathrm{w}}\right)$ and one associated with the strong interaction $\left(k_{\mathrm{s}}\right)$ might be sufficient. The number of fundamental physical constants equals the number of fundamental physical notions, such as space, time, mass and quantized charges, which allows to use those constants as generators of the fundamental base units. There is no one-to-one correspondence between the fundamental constants and the fundamental notions, as those notions are interdependent. Fundamental physical constants are true scalars, i.e. they are invariant under any transformation.

The Boltzmann constant $k_{\mathrm{B}}$ and the Avogadro constant $N_{\mathrm{A}}$ are just "physical constants" or "conventional physical constants". The two constants are true scalars and generators of the kelvin and the mole, respectively. Although $k_{\mathrm{B}}, N_{\mathrm{A}}$ and the corresponding units are very important and convenient for practical use, they are not fundamental, as they are not indispensable to handle description of physical phenomena and (in principle) one could do without both of them.

In this paper, physical properties of microscopic entities (molecules, atoms, elementary particles or fundamental particles) are considered "microscopic material constants" or "microscopic material parameters" (as those are constant under certain conditions). Properties of elementary or fundamental particles (e.g. the rest mass and the charge of the proton or the electron) are distinguished from fundamental physical constants, because (1) their effective values may vary at very high energies due to their internal structure and/or external structure (vacuum polarization); and (2) elemental physical phenomena (relativistic effects, quantum effects or gravity) do not necessitate a reference to those particles and their properties.

Fundamental particles are such that do not comprise any internal constituent entities; these are leptons, quarks and interaction bosons (i.e. the photon, weak and strong interaction gauge bosons and the hypothetical graviton). Elementary particles are built of one or more fundamental particles.

Fundamental interactions are: gravity, electromagnetic, weak and strong interactions, irrespective of our ability to describe them in a unified way (either in terms of the electroweak theory, the Standard Model or the Grand Unified Theory).

Unification of interactions (without the "GUT" qualifier) means that two or more fundamental interactions have been accounted for in one theory, which nevertheless preserves identity of those interactions (similarly as atoms preserve their identity within a molecule). Such unification does not change the number of fundamental interactions and the number of the corresponding coupling constants. Unification may produce relations between coupling constants of constituent interactions, but the overall number of independent constants does not change (e.g., the weak coupling constant is related to the electromagnetic coupling constant, but another independent constant is simultaneously introduced - the Wein- 
berg angle $\theta$ ). The "GUT unification" may (although does not have to) fuse all four fundamental interactions into one, with only one coupling constant.

Coupling constants are fundamental constants that characterize strength of fundamental interactions. There is one-to-one correspondence between fundamental interactions and their coupling constants. Coupling constants of quantized interactions are true scalars and generators of fundamental base units for the corresponding charges. The gravitational coupling constant $G$ is of a distinct physical character, because gravity is not quantized; the $G$ is also a true scalar and together with $h$ and $c$ form a set of three fundamental constants that define the base units of time, distance and mass.

There is only one candidate for the gravitational coupling constant, the $G$. The choice of coupling constants for quantized interactions is not unique. For example, the coupling constant of the electromagnetic interaction can be: the elementary charge $e$ (in particle physics), the fine structure constant $\alpha$ (in atomic physics), or $k_{\mathrm{e}}=1 /\left(4 \pi \varepsilon_{0}\right)$ - in the traditional approach and in this paper. Each choice is fine, as long as it is used consistently.

The elementary charge $e$ is a constant, irrespective of whether it is considered the coupling constant and its value is fixed by definition or not. The $e$ cannot be treated as identical with the electric charge of a certain particle, but (for practical purposes) it equals the magnitude of the electric charge of the dressed electron.

In symbolic calculations, however, one may consider both the $e$ and the $k_{\mathrm{e}}$ (as well as $\varepsilon_{0}$ and $\mu_{0}$ ) as exactly known constants; one has to be careful, however, when numerical values are computed and the uncertainty is to be evaluated. One can fix the value of either of those two constants: (1) the $e$ may be given a fixed value (as in the New SI) and then the $k_{\mathrm{e}}$ becomes a measurable quantity; or (2) one may fix the value of $k_{\mathrm{e}}$ (as in the FC SI), or fix the value of an equivalent constant (such as $\varepsilon_{0}$ or $\mu_{0}$ in the present $\mathrm{SI}$ ), and then the $e$ is a measurable quantity. However, one cannot fix values of both quantities simultaneously, neither explicitly, nor implicitly, because those constants would then be overconstrained.

\section{References}

[1] B.N. Taylor, P.J. Mohr, Metrologia 36, 63 (1999).

[2] I.M. Mills, P.J. Mohr, T.J. Quinn, B.N. Taylor, E.R. Williams, Metrologia 42, 71 (2005).

[3] J.L. Flowers, B.W. Petley, Metrologia 42, L31 (2005).

[4] R. Davis, in: IMEKO 20th TC3, 3rd TC16 and 1st TC22 Int. Conf. "Cultivating metrological knowledge", Merida (Mexico), 2007, IMEKO webside.

[5] J.W.G. Wignall, Meas. Sci. Technol. 16, 682 (2005).

[6] B.P. Leonard, Metrologia 44, L4 (2007).

[7] B.P. Kibble, in: Atomic Masses and Fundamental Constants, Vol. 5, Eds. J.H. Sanders, A.H. Wapstra, Plenum, New York 1975, p. 545.

[8] B.P. Kibble, I.A. Robinson, Meas. Sci. Technol. 14, 1243 (2003)
[9] R. Steiner, D. Newell, E. Williams, J. Res. Natl. Inst. Stand. Technol. 110, 1 (2005).

[10] R.L. Steiner, E.R. Williams, D.B. Newell, R. Liu, Metrologia 42, 431 (2005).

[11] I.A. Robinson, B.P. Kibble, Metrologia 44, 427 (2007).

[12] A. Eichenberger, B. Jeckelmann, P. Richard, Metrologia 40, 356 (2003).

[13] A. Eichenberger, G. Genevès, P. Gournay, Eur. Phys. J. Special Topics 172, 363 (2009).

[14] T.J. Quinn, Metrologia 37, 87 (2000).

[15] A. Wallard, Metrologia 43, 175 (2006).

[16] I.M. Mills, P.J. Mohr, T.J. Quinn, B.N. Taylor, E.R. Williams, Metrologia 43, 227 (2006).

[17] I. Mills, Draft Chapter 2 for SI Brochure, following redefinitions of the base units, CCU Report, 27th September 2010, BIPM website, 14 pages (2010).

[18] J.C. Maxwell, Address to the Mathematical and Physical Sections of the British Association, Liverpool, Sept. 15, 1870; reprint in Maxwell on Molecules and Gases, Eds. E. Garber, S.G. Brush, C.W.F. Everitt, MIT, Cambridge 1986, p. 89.

[19] Resolution 6 of the 11th meeting of the CGPM (1960), in: SI Brochure, 8th ed., p. 148.

[20] Resolution 1 of the 17th meeting of the CGPM (1983), Metrologia 20, 25 (1984).

[21] P. Becker, P. De Bièvre, K. Fujii, M. Glaeser, B. Inglis, H. Luebbig, G. Mana, Metrologia 44, 1 (2007).

[22] A. Wallard, Metrologia 47, 103 (2010).

[23] B.W. Petley, Metrologia 44, 69 (2007).

[24] H. Harari, N. Seiberg, Phys. Lett. B 98, 269 (1981).

[25] P. Schmüser, H. Spitzer, in: Constituents of Matter. Atoms, Molecules, Nuclei and Particles, Ed. W. Raith and series Eds. L. Bergmann, C. Schaefer, Walter de Gruyter, Berlin 1997.

[26] J.J. Sakurai, Advanced Quantum Mechanics, 10th ed., Benjamin-Cummings, Menlo Park, CA 1984.

[27] C. Itzykson, J.B. Zuber, Quantum Field Theory, McGraw-Hill, New York 1980.

[28] H.B.G. Casimir, D. Polder, Phys. Rev. 73, 360 (1948).

[29] M. Bordag, G.L. Klimchitskaya, U. Mohideen, V.M. Mostepanenko, Advances in the Casimir Effect, Oxford University Press, Oxford 2009.

[30] M.J. Sparnaay, Physica 24, 751 (1958).

[31] SLAC Press release 06/28/2005 SLAC experiment makes first observations of key traits in weak force, Stanford (2005); http://home.slac.stanford.edu/ pressreleases/2005/20050628.htm .

[32] M. Schmelling, in: XXVIII Int. Conf. High Energy Physics, Warsaw, July 25-31 (1996); arXiv:hep-ex/ 9701002v1.

[33] S. Weinberg, The Quantum Theory of Fields, Vol. 2, Modern Applications, Cambridge University Press, Cambridge 1996.

[34] K. Huang, Statistical Mechanics, 2nd ed., Wiley, New York 1987.

[35] A.L. Fetter, J.D. Walecka, Quantum Theory of Many-Particle Systems, Mc Graw-Hill, New York 1971. 
[36] W.T. Chyla, Can. J. Phys. 78, 721 (2000).

[37] M. Michelini, Prog. Phys. 3, 64 (2009).

[38] Special section, Eds. C.C. Speake, T.J. Quinn: Meas. Sci. Technol. 10, 419 (1999).

[39] P.J. Mohr, D.B. Newell, Am. J. Phys. 78, 338 (2010).

[40] T.J. Quinn, C.C. Speake, S.J. Richman, R.S. Davis, A. Picard, Phys. Rev. Lett. 87, 111101 (2001).

[41] S. Schlamminger, E. Holzschuh, W. Kündig, F. Nolting, R.E. Pixley, J. Schurr, U. Straumann, Phys. Rev. D 74, 082001 (2006).

[42] A.D. Alexeev, K.A. Bronnikov, N.I. Kolosnitsyn, M.Y. Konstantinov, V.N. Melnikov, A.J. Sanders, Metrologia 38, 397 (2001).
[43] J.B. Fixler, G.T. Foster, J.M. McGuirk, M.A. Kasevich, Science 315, 74 (2007).

[44] G. Lamporesi, A. Bertoldi, L. Cacciapuoti, M. Prevedelli, G.M. Tino, Phys. Rev. Lett. 100 , 050801 (2008).

[45] F. Cabiati, W. Bich, Metrologia 46, 457 (2009).

[46] P.J. Mohr, Metrologia 45, 129 (2008).

[47] M.J. Duff, L.B. Okun, G. Veneziano, J. High Energy Phys. 03, 023 (2003). 